\title{
Characterization of crystalline dendrimer-stabilized gold nanoparticles
}

\author{
Xiangyang Shi ${ }^{1}$, T Rose Ganser ${ }^{1}$, Kai Sun ${ }^{2}$, Lajos $\mathbf{P}$ Balogh $^{1}$ and \\ James R Baker Jr $\mathbf{~}^{1}$ \\ ${ }^{1}$ Michigan Nanotechnology Institute for Medicine and Biological Sciences, University of \\ Michigan, Ann Arbor, MI 48109-0648, USA \\ 2 Electron Microbeam Analysis Laboratory, 413 B Space Research Building, University of \\ Michigan, Ann Arbor, MI 48109-2143, USA \\ E-mail: jbakerjr@umich.edu
}

Received 12 October 2005, in final form 13 December 2005

Published 30 January 2006

Online at stacks.iop.org/Nano/17/1072

\begin{abstract}
Monodispersed, highly crystalline dendrimer-stabilized gold nanoparticles (Au DSNPs) were synthesized via hydrazine reduction chemistry and stabilized using primary amine-terminated poly(amidoamine) (PAMAM) dendrimers of different generations (generations 2-6) with the same molar ratios of dendrimer terminal nitrogen ligands/gold atoms. The sizes of the synthesized Au DSNPs decrease with the increase of the number of dendrimer generations. These Au DSNPs are fluorescent and display strong blue emission intensity at $458 \mathrm{~nm}$. Polyacrylamide gel electrophoresis (PAGE) analysis indicates that all Au DSNPs are stable and both metal NPs and dendrimer stabilizers do not separate from each other during the electrophoresis process. The synthesized inorganic/organic hybrid $\mathrm{Au}$ DSNPs provide new nanoplatforms that will be further modified with various biological ligands for the application of biosensing and targeted cancer therapeutics.
\end{abstract}

(Some figures in this article are in colour only in the electronic version)

\section{Introduction}

Recent advances in biologic nanotechnology show that $\mathrm{Au}$ nanoparticles (NPs) can be used as platforms for biodiagnosis, biosensing, gene delivery carriers, and targeted drug delivery [1-4]. In most cases, the surface modification and engineering of Au NPs has to be performed before they are applied in biological systems. For instance, peptides $[1,5,6]$ and proteins $[7,8]$ can be conjugated onto Au NPs for targeting purposes; antibody has been conjugated onto $\mathrm{Au}$ NP surfaces for immunoassay and biosensing [9, 10]; DNAmodified Au NPs can be used as nanoprobes to detect target DNA sequences through hybridization $[2,4]$. The Au NPs used for bioconjugation were commonly prepared by citric acid reduction and protection under an elevated temperature. Thio chemistry has been extensively utilized in the conjugation of Au NPs with various biological ligands $[2,3]$.

An alternative approach to prepare metal NPs has been developed by using poly(amidoamine) (PAMAM) dendrimers as templates or stabilizers [11-14]. PAMAM dendrimers are close to spherical, highly branched macromolecules with symmetrically emanating dendrons of defined molecular weight and size. They are composed of a core molecule and dendritic branches that regularly extend from the core to terminal groups [15-17]. PAMAMs are ideal macromolecules with a narrow polydispersity to encapsulate and stabilize metal NPs due to their 'built-in' functional groups, fairly uniform composition and defined structures. The formed organic/inorganic hybrid metal dendrimer NPs hold great promise in various applications such as catalysis [18], optics [19, 20], biological sensing [21], cancer therapeutics [22], and building blocks to assemble functional films [23, 24]. The preparation of dendrimer-stabilized Au NPs (Au DSNPs) usually involves complexation of gold salts (e.g. $\mathrm{HAuCl}_{4}$ ) with PAMAM dendrimers, followed by physical or chemical reduction [13, 25-27]. Several groups pioneered the research on dendrimer encapsulated/stabilized metal NPs [11, 12, 14] as well as specifically on Au DSNPs [12, 27-29]. It is well 
Characterization of crystalline dendrimer-stabilized gold nanoparticles

Table 1. Preparation parameters and zeta potentials of Au DSNPs.

\begin{tabular}{lllllllll}
\hline Au DSNPs & $\mathrm{D}^{\mathrm{a}}$ & $\begin{array}{l}C_{\mathrm{D}} \\
\left(\mathrm{mg} \mathrm{ml}^{-1}\right)\end{array}$ & $\begin{array}{l}\mathrm{D} \\
(\mathrm{mol})\end{array}$ & $\begin{array}{l}\mathrm{Au} \\
(\mathrm{mol})\end{array}$ & $\begin{array}{l}C_{\text {Au }} \\
\left(\mathrm{mg} \mathrm{ml}^{-1}\right)\end{array}$ & $\begin{array}{l}\text { Au:D } \\
\text { molar ratio }\end{array}$ & $\begin{array}{l}\mathrm{DTA}^{\mathrm{b}} / \mathrm{Au} \\
\text { molar ratio }\end{array}$ & $\begin{array}{l}\text { Zeta potential } \\
(\mathrm{mV})\end{array}$ \\
\hline$\left\{\left(\mathrm{Au}^{0}\right)_{6}-\mathrm{E} 2 . \mathrm{NH}_{2}\right\}$ & $\mathrm{E} 2 . \mathrm{NH}_{2}$ & 10.82 & $1.6610 \mathrm{e}-5$ & $9.95 \mathrm{e}-5$ & 0.003940 & $5.99: 1$ & $2.67: 1$ & +39.67 \\
$\left\{\left(\mathrm{Au}^{0}\right)_{12}-\mathrm{E} 3 . \mathrm{NH}_{2}\right\}$ & $\mathrm{E} 3 . \mathrm{NH}_{2}$ & 11.29 & $8.1710 \mathrm{e}-6$ & $9.95 \mathrm{e}-5$ & 0.003940 & $12.18: 1$ & $2.63: 1$ & +26.42 \\
$\left\{\left(\mathrm{Au}^{0}\right)_{24}-\mathrm{E} 4 . \mathrm{NH}_{2}\right\}$ & $\mathrm{E} 4 . \mathrm{NH}_{2}$ & 11.90 & $4.1860 \mathrm{e}-6$ & $9.95 \mathrm{e}-5$ & 0.003940 & $23.78: 1$ & $2.69: 1$ & +39.98 \\
$\left\{\left(\mathrm{Au}^{0}\right)_{57}-\mathrm{E}_{2} \cdot \mathrm{NH}_{2}\right\}$ & $\mathrm{E} 5 . \mathrm{NH}_{2}$ & 10.14 & $1.7580 \mathrm{e}-6$ & $9.95 \mathrm{e}-5$ & 0.003940 & $56.61: 1$ & $2.26: 1$ & +41.11 \\
$\left\{\left(\mathrm{Au}^{0}\right)_{98}-\mathrm{E}_{2} \cdot \mathrm{NH}_{2}\right\}$ & $\mathrm{E} 6 . \mathrm{NH}_{2}$ & 11.77 & $1.0140 \mathrm{e}-6$ & $9.95 \mathrm{e}-5$ & 0.003940 & $98.15: 1$ & $2.61: 1$ & +40.21 \\
\hline
\end{tabular}

${ }^{a} \mathrm{D}$ denotes dendrimer.

${ }^{\mathrm{b}}$ DTA denotes dendrimer terminal amines.

established that the size of the Au DSNPs is mainly dependent on the molar ratio between dendrimers and $\mathrm{Au}$ atoms [25]. The mechanistic studies show that dendrimer terminal amines are extremely effective in the stabilization of Au NPs [28, 30]. Although extensive studies have been performed regarding their synthesis, characterization, and potential applications, the systematic studies of generation-dependent structure and properties of Au DSNPs using systematic characterization techniques are still not fully investigated. Complete understanding of the formed nanohybrid structures from different aspects of view still remains a great challenge $[29,30]$.

In this work, we selected primary amine-terminated PAMAMs of generations 2-6 to prepare Au DSNPs with consistent molar ratios of dendrimer terminal amines and gold atoms. Various characterization techniques were employed to investigate their structural characteristics, including UVvis spectrometry, fluorescence spectroscopy, transmission electron microscopy (TEM), zeta potential measurements, and polyacrylamide gel electrophoresis (PAGE). UV-vis and fluorescence spectrometry disclose the optical properties of Au DSNPs, while electron microscopy imaging and selected area electron diffraction (SAED) characterize the morphology, sizes, and crystal structure of Au DSNPs. Zeta potential measurements were used to record the surface charges of the formed Au DSNPs. The stability of Au DSNPs was further characterized using PAGE. The presented studies shed light on the understanding of the structures and properties of Au DSNPs, which is helpful for us to further post-modify them with various biological ligands for biological sensing, targeting, and treatment of cancers.

\section{Experimental section}

\subsection{Materials}

Amine-terminated PAMAM dendrimers of generations 2-6 (E2. $\mathrm{NH}_{2}-\mathrm{E} 6 . \mathrm{NH}_{2}$; E denotes the ethylenediamine core; the number denotes the generation; the same nomenclature is applied to all other dendrimers used in this work) were purchased from Dendritech (Midland, MI, USA). Gold colloids with diameters of 5 and $100 \mathrm{~nm}$ were from Ted Pella (Redding, CA, USA). All other chemicals were obtained from Aldrich and used as received. Water used in all of the experiments was purified using a Milli-Q Plus 185 water purification system (Millipore, Bedford, MA, USA) with resistivity higher than $18 \mathrm{M} \Omega \mathrm{cm}$. Regenerated cellulose dialysis membranes $(\mathrm{MWCO}=10000)$ were acquired from Fisher.

\subsection{Synthesis of $A u D S N P s$}

The Au DSNPs were prepared using hydrazine reduction chemistry in the same dendrimer terminal amine (DTA)/gold atom molar ratios according to the described procedure in the literature [27]. They are denoted as $\left\{\left(\mathrm{Au}^{0}\right)_{6}-\mathrm{E} 2 \cdot \mathrm{NH}_{2}\right\}$, $\left\{\left(\mathrm{Au}^{0}\right)_{12}-\mathrm{E} 3 . \mathrm{NH}_{2}\right\}, \quad\left\{\left(\mathrm{Au}^{0}\right)_{24}-\mathrm{E} 4 . \mathrm{NH}_{2}\right\}, \quad\left\{\left(\mathrm{Au}^{0}\right)_{57}-\mathrm{E} 5 . \mathrm{NH}_{2}\right\}$, and $\left\{\left(\mathrm{Au}^{0}\right)_{98}-\mathrm{E} 6 . \mathrm{NH}_{2}\right\}$, respectively. Note that the numbers of $6,12,24,57$, and 98 are the numbers of $\mathrm{Au}$ atoms per dendrimer molecule according to the preparation stoichiometry (table 1). The denotation of $\left\{\left(\mathrm{Au}^{0}\right)_{x}-\mathrm{E} n \cdot \mathrm{NH}_{2}\right\}$ (where $x$ is the number of $\mathrm{Au}$ atoms and $n$ the number of the dendrimer generation) does not necessarily represent one single particle. Briefly, gold-dendrimer complexes were prepared in aqueous solution by mixing $5 \mathrm{ml}$ of $20 \mathrm{mM}$ aqueous solutions of $\mathrm{HAuCl}_{4}$ with $5 \mathrm{ml}$ aqueous solutions of the respective PAMAM dendrimers with identical molar ratios of DTA/Au atoms. The yellow $\mathrm{HAuCl}_{4}$ solution lost its colour immediately upon mixing with the PAMAMs, indicating the formation of complexes between the dendrimer terminal amines and the gold anions. Stable Au DSNPs were prepared by reducing the PAMAM-tetrachloroaurate complexes at room temperature with $50 \mathrm{~mol} \%$ excess of hydrazine under vigorous magnetic stirring for $2 \mathrm{~h}$. Upon addition of the hydrazine solution to the PAMAM-tetrachloroaurate complexes, a colour change from slightly yellow to deep red indicated the formation of zero-valent gold.

\subsection{UV-vis spectrometry}

UV-vis spectra were collected using a Lambda $20 \mathrm{UV}-\mathrm{vis}$ spectrometer. All Au DSNP samples were dissolved in water at the concentration of $0.1 \mathrm{mg} \mathrm{ml}^{-1}$. For comparison, all dendrimer samples in the absence of Au NPs at a concentration of $0.1 \mathrm{mg} \mathrm{ml}^{-1}$ were also measured.

\subsection{Fluorescence spectroscopy}

The excitation and emission spectra of Au DSNPs were collected using the Fluoromax-2 fluorimeter. All samples were dissolved in water at the concentration of $0.1 \mathrm{mg} \mathrm{ml}^{-1}$. The maximum emission and excitation wavelengths were used for collecting excitation and emission spectra, respectively. Both excitation and emission slit openings were set as $5 \mathrm{~nm}$. In order to determine the source of fluorescence for Au DSNP samples, excitation and emission spectra of dendrimer stabilizers were also measured using their maximum emission and excitation wavelengths under the same conditions. 


\subsection{Zeta potential measurements}

Zeta potential measurements were performed using a PSS/NICOMP 380 ZLS particle sizing system (Santa Barbara, CA) with a red-diode laser at $635 \mathrm{~nm}$ in a multiangle cell.

\subsection{TEM}

A JEOL 2010F analytical electron microscope was performed at $200 \mathrm{kV}$ with an energy dispersive spectroscopy (EDS) system attached. $5 \mu \mathrm{l}$ aqueous solution of Au DSNPs $\left(0.1 \mathrm{mg} \mathrm{ml}^{-1}\right)$ was dropped onto a carbon-coated copper grid and air dried before measurements. Ultrathin sections of the PAGE gel samples of Au-DSNPs for TEM were sliced with a Leica Ultracut UCT ultramicrotome after setting them in LRwhite resin. The thin sections were placed onto carbon-coated copper grids before TEM imaging.

\subsection{PAGE}

Analysis of PAMAM dendrimers and Au DSNPs by PAGE was performed on a Micrograd vertical electrophoresis system (model FB-VE10-1, FisherBiotech, Pittsburgh, PA, USA) with a commercial power supply (model EC135-90; Thermo Electron Corporation, Milford, MA, USA). Precast 4-20\% gradient express gels for PAGE were obtained from ISC BioExpress (Kaysville, UT, USA). Tris-glycine native buffer $(\mathrm{pH}=8.3$ ) was purchased from Invitrogen (Carlsbad, CA, USA). It was diluted 10 times to prepare the running buffer. PAGE separations typically required $50 \mathrm{~min}$ at $200 \mathrm{~V}$. Reverse polarity was used for the analysis of the polycationic PAMAM dendrimers and $\mathrm{Au}$ DSNPs. Into each sample well $2 \mu \mathrm{l}$ of a sample solution composed of $1 \mu 11 \mathrm{mg} \mathrm{m}^{-1}$ PAMAM dendrimer or $\mathrm{Au}$ DSNPs and $1 \mu \mathrm{l}$ methylene blue sucrose dye solutions (50\% sucrose, $1 \%$ methylene blue) was injected. Developed gels were stained with $0.025 \%$ Coomassie blue R250 in $40 \%$ methanol and $7 \%$ acetic acid aqueous solution overnight. The gels were destained with an aqueous solution containing $7 \%(\mathrm{v} / \mathrm{v})$ acetic acid and $5 \%(\mathrm{v} / \mathrm{v})$ methanol.

\section{Results and discussion}

The stoichiometry used to prepare Au DSNPs is listed in table 1. For all Au-DSNPs, the molar ratios between dendrimer terminal amines (DTAs) and Au atoms are consistent except that there is a little variation for the $\mathrm{E} 5 . \mathrm{NH}_{2}$ dendrimer. Figure 1(a) shows the UV-vis spectra of Au DSNPs prepared using E2. $\mathrm{NH}_{2}$ through E6. $\mathrm{NH}_{2}$. The plasmon peak at around $525 \mathrm{~nm}$, which is attributed to collective oscillation of free electrons in gold NPs [31, 32], is clearly observed for all samples. Spherical particles with diameters less than several hundred nanometres exhibit a single, strong absorption peak in the visible region of the spectrum [33], which is called the surface plasmon peak. The gradual increase of absorption below $400 \mathrm{~nm}$ for all samples is due to the interband transition of gold. The absorbance peak at $283 \mathrm{~nm}$ for all Au DSNPs is assigned to certain carbonyl compounds formed presumably by oxidation of the dendrimers [12]. The same UV-vis spectrometric features related to Au DSNPs were also observed by other groups [12, 27]. A recent report from Bard's group clearly shows that PAMAM dendrimers oxidized by
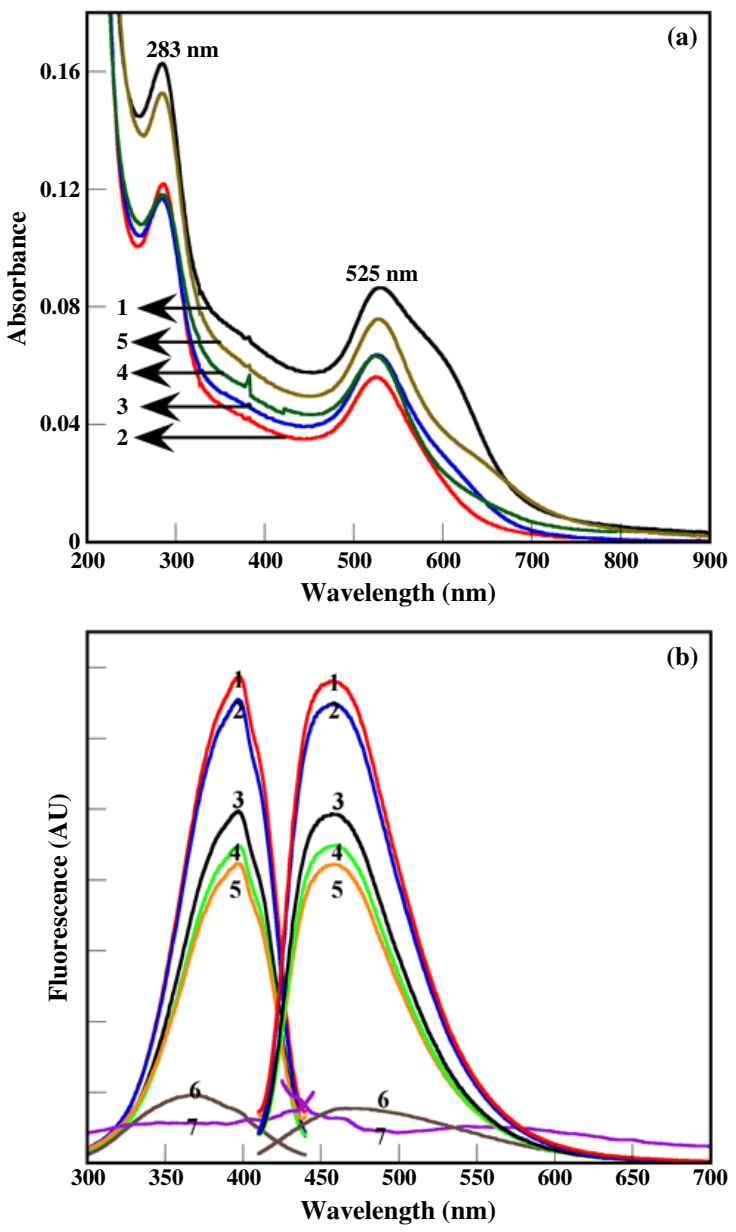

Figure 1. UV-vis (a) and fluorescence (b) spectra of Au DSNPs. Curves $1,2,3,4$, and 5 correspond to $\left\{\left(\mathrm{Au}^{0}\right)_{6}-\mathrm{E}_{2} \cdot \mathrm{NH}_{2}\right\}$, $\left\{\left(\mathrm{Au}^{0}\right)_{12}-\mathrm{E} 3 . \mathrm{NH}_{2}\right\},\left\{\left(\mathrm{Au}^{0}\right)_{24}-\mathrm{E} 4 \cdot \mathrm{NH}_{2}\right\},\left\{\left(\mathrm{Au}^{0}\right)_{57}-\mathrm{E} 5 . \mathrm{NH}_{2}\right\}$, and $\left\{\left(\mathrm{Au}^{0}\right)_{98}-\mathrm{E} 6 . \mathrm{NH}_{2}\right\}$, respectively. In (b), 6 and 7 indicate gold colloids with diameters of 5 and $100 \mathrm{~nm}$, respectively.

$\left(\mathrm{NH}_{4}\right)_{2} \mathrm{~S}_{2} \mathrm{O}_{8}$ or Au salts display a strong UV-vis absorbance at $290 \mathrm{~nm}$ [34]. We believe that the absorbance of Au DSNPs at $283 \mathrm{~nm}$ is from dendrimers which are oxidized by Au salts during the formation of Au DSNPs [34, 35]. In contrast, PAMAM dendrimers themselves without oxidation by Au salts do not display any absorption features at wavelength above $250 \mathrm{~nm}$ (figure 2(a)).

Figure 1(b) shows the fluorescence spectra of Au DSNPs and commercial Au colloid particles (5 and $100 \mathrm{~nm}$ ). All Au DSNPs were found to be fluorescent and display strong blue photoluminescence. The maximum excitation and emission wavelengths were around 397 and $458 \mathrm{~nm}$, respectively, in agreement with the literature data [36]. In contrast, commercial gold colloids (5 and $100 \mathrm{~nm}$ ) that are prepared using a citric acid reduction and protection approach do not exhibit fluorescence emission, suggesting that the dendrimer stabilizers contribute to the fluorescence properties of the formed Au DSNPs. A recent report from Imae's group shows that both PAMAM and polypropyleneimine (PPI) dendrimers exhibit strong intrinsic fluorescence emission at certain concentration ranges [37]. They proposed that the backbone of dendrimers plays a key role in forming the 

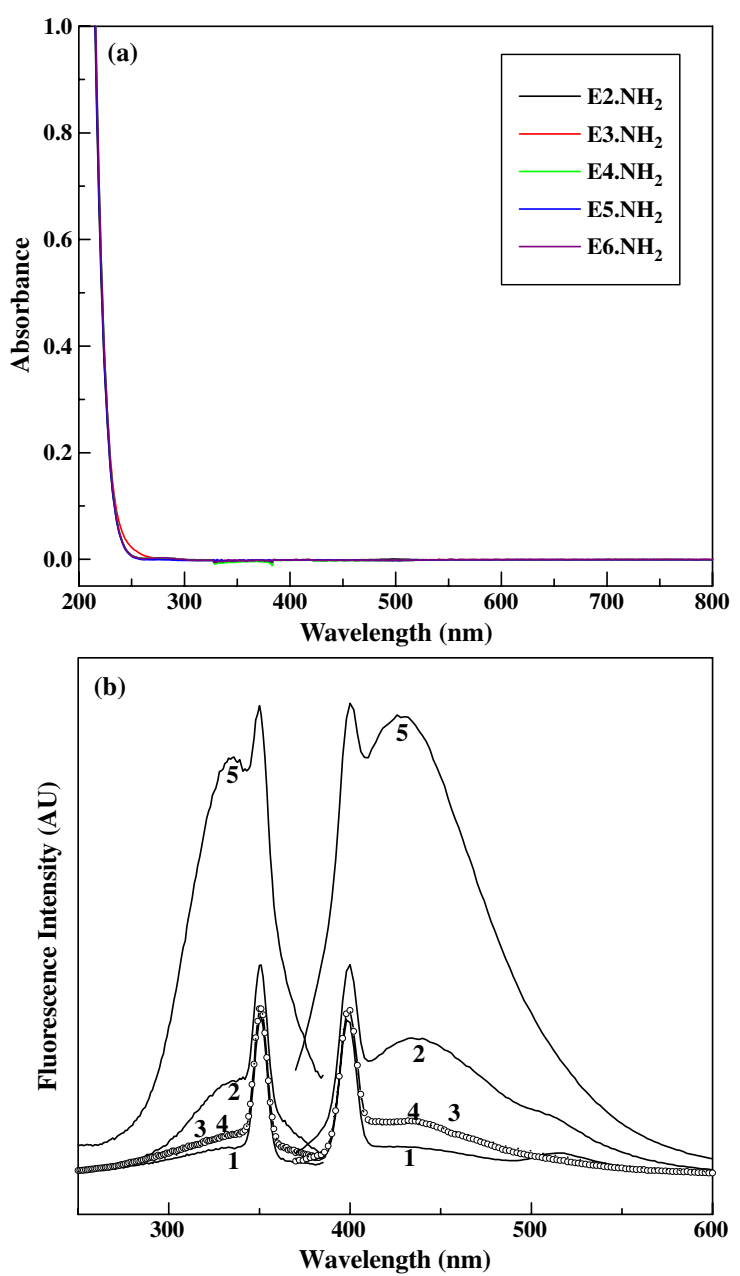

Figure 2. UV-vis (a) and fluorescence (b) spectra of dendrimer stabilizers. In (b), curves 1, 2, 3, 4 and 5 correspond to E2. $\mathrm{NH}_{2}$, E3. $\mathrm{NH}_{2}, \mathrm{E} 4 . \mathrm{NH}_{2}, \mathrm{E} 5 . \mathrm{NH}_{2}$ and E6. $\mathrm{NH}_{2}$, respectively.

fluorescence centre. For comparison, we also investigated the fluorescence properties of dendrimer stabilizers in the absence of Au NPs (figure 2(b)). It is clear that dendrimer stabilizers of different generations exhibit intrinsic blue photoluminescence. The maximum excitation and emission wavelengths for all dendrimer stabilizers $\left(\mathrm{E} 2 . \mathrm{NH}_{2}\right.$ to $\left.\mathrm{E} 6 . \mathrm{NH}_{2}\right)$ are around 350 and $400 \mathrm{~nm}$, respectively. The slight variation of maximum excitation and emission wavelength between Au DSNPs and dendrimer stabilizers may be related to the strong interaction between Au nanocrystals and dendrimer terminal amines. It is interesting to note that the excitation spectra of dendrimer stabilizers are really different from those UV-vis spectra of the respective dendrimers (figure 2). The maximum excitation wavelengths at $350 \mathrm{~nm}$ for dendrimers of different generations do not appear in their corresponding UV-vis spectra. Generally, the UV-vis spectrum of a small organic molecule is always expected to be similar to its excitation fluorescence spectrum. However, this is not the case for PAMAM dendrimers due to their complex structures. The same situation and explanation also applies to Au DSNPs regarding the difference between their UV-vis and excitation fluorescence spectra (figure 1). We also note that although Au DSNPs display a complete reverse order of the relative fluorescence intensity as a function of the number of the dendrimer generation (figure 1(b)), the dendrimer stabilizers do not show a regular relationship in the similar case scenario (figure 2(b)). In all cases, the Au DSNPs and dendrimer stabilizers are different materials that do not necessarily follow a strict correlation in terms of their relative fluorescence intensities as a function of the dendrimer generation. The fluorescent properties of the formed Au DSNPs make them potentially useful as fluorescent markers for cell labelling and biological sensing studies.

The size distribution and morphology of the synthesized Au DSNPs were studied by TEM. Figure 3 shows TEM images of Au DSNPs prepared using PAMAM dendrimers of different generations. The sizes of the formed $\mathrm{Au}$ DSNPs are $15.4 \pm 5.8 \mathrm{~nm}, 12.0 \pm 2.8 \mathrm{~nm}, 9.1 \pm 3.2 \mathrm{~nm}$, $8.6 \pm 2.8 \mathrm{~nm}$, and $7.1 \pm 1.9 \mathrm{~nm}$ for $\left\{\left(\mathrm{Au}^{0}\right)_{6}-\mathrm{E} 2 . \mathrm{NH}_{2}\right\}$, $\left\{\left(\mathrm{Au}^{0}\right)_{12}-\mathrm{E} 3 . \mathrm{NH}_{2}\right\}, \quad\left\{\left(\mathrm{Au}^{0}\right)_{24}-\mathrm{E} 4 . \mathrm{NH}_{2}\right\}, \quad\left\{\left(\mathrm{Au}^{0}\right)_{57}-\mathrm{E} 5 . \mathrm{NH}_{2}\right\}$, and $\left\{\left(\mathrm{Au}^{0}\right)_{98}-\mathrm{E} 6 . \mathrm{NH}_{2}\right\}$, respectively. It is clear that all the Au DSNPs are relatively monodispersed except $\left\{\left(\mathrm{Au}^{0}\right)_{6}\right.$ E2. $\left.\mathrm{NH}_{2}\right\} . \quad\left\{\left(\mathrm{Au}^{0}\right)_{6}-\mathrm{E} 2 . \mathrm{NH}_{2}\right\}$ displays larger size and higher polydispersity, which is attributed to the limited number of amines of the E2. $\mathrm{NH}_{2}$ dendrimer to stabilize Au NPs. This result is in good agreement with the reported data [38]. The size of the Au DSNPs decreases with the increase of the number of dendrimer generations (figure 3(f)), suggesting different nucleation and growth mechanisms for gold nanocrystals in the presence of PAMAM dendrimers. At basic $\mathrm{pH}$ conditions $(\mathrm{pH} \approx 10.4$ when dendrimers are dissolved in water), $\mathrm{AuCl}_{4}^{-}$anions bind preferably to the protonated amines of PAMAM dendrimers through electrostatic interaction. Higher generation PAMAM dendrimers have denser structures that would significantly limit the nucleation, movement, and growth of gold nanocrystals. In contrast, lower generation PAMAMs have relatively open structures, which hinder the growth of gold nanocrystals less significantly than higher generation PAMAMs. It is interesting to note that the synthesized Au DSNPs in this work are significantly larger than those reported in the literature $[29,30,38,39]$. It is known that the NP sizes and morphologies are variable under different preparation conditions such as selection of reduction reagents, concentration, temperature, and solvent systems [40]. It is believed that fast reduction always induces the formation of smaller NPs, while slow reduction favours the aggregation and Ostwald ripening of NPs [41]. The reduction potential of $\mathrm{NH}_{2}=\mathrm{NH}_{2}(-0.09 \mathrm{~V})$ used in this work is significantly smaller than $\mathrm{NaBH}_{4}(-0.481 \mathrm{~V})$, which was used by other groups $[42,43]$. Therefore, the slower reaction rate favours the formation of larger Au DSNPs; accordingly, the formed Au DSNPs are covered with a monolayer of dendrimer molecules. In contrast, dendrimer-encapsulated Au NPs with diameter less than $5 \mathrm{~nm}$ can be prepared by a fast reduction process using $\mathrm{NaBH}_{4}[29,38,39]$. In the latter case, the dendrimer itself serves as a template to encapsulate an individual Au NP. Compared with dendrimer-encapsulated Au NPs synthesized using $\mathrm{NaBH}_{4}$ reduction chemistry, the size dispersity of $\mathrm{Au}$ DSNPs is rather large, mainly due to the fact that the slow nucleation process of NPs occurs because of the slow reduction reaction. However, the size dispersity of Au DSNPs synthesized using hydrazine reduction chemistry is comparable with those synthesized under UV or laser radiation [12, 41]. 


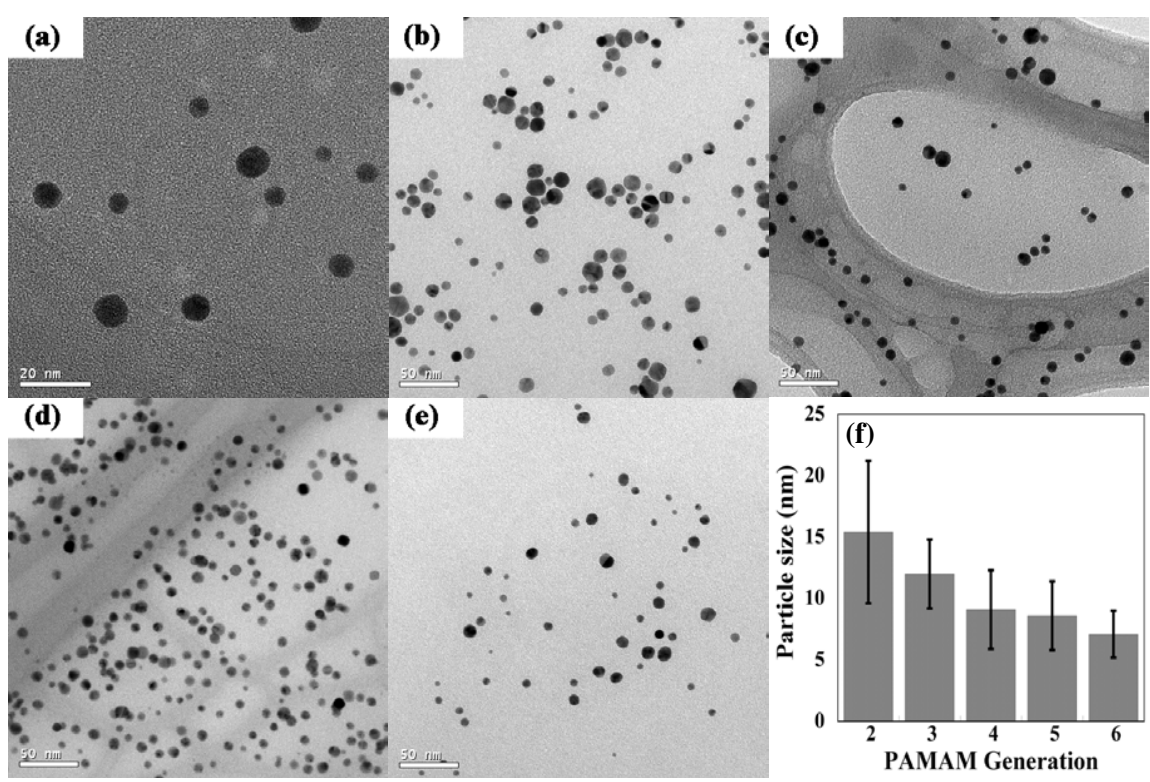

Figure 3. Large scale TEM images of (a) $\left\{\left(\mathrm{Au}^{0}\right)_{6}-\mathrm{E} 2 \cdot \mathrm{NH}_{2}\right\}$, (b) $\left\{\left(\mathrm{Au}^{0}\right)_{12}-\mathrm{E} 3 \cdot \mathrm{NH}_{2}\right\}$, (c) $\left\{\left(\mathrm{Au}^{0}\right)_{24}-\mathrm{E} 4 \cdot \mathrm{NH}_{2}\right\}$, (d) $\left\{\left(\mathrm{Au}^{0}\right)_{57}-\mathrm{E} 5 \cdot \mathrm{NH}_{2}\right\}$, and (e) $\left\{\left(\mathrm{Au}^{0}\right)_{98}-\mathrm{E} 6 . \mathrm{NH}_{2}\right\}$. The plot of their size as a function of the number of dendrimer generations is shown in (f).

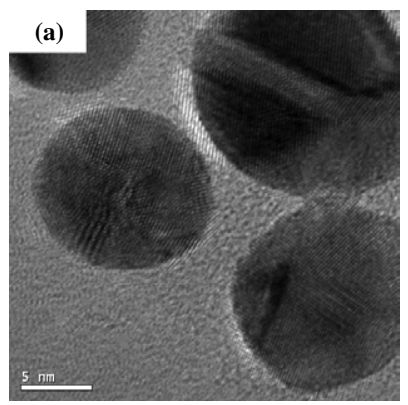

(c)
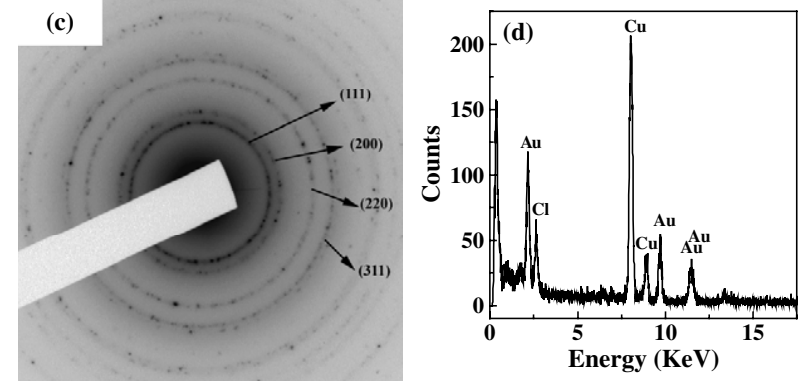

Figure 4. High-resolution TEM images of $\left\{\left(\mathrm{Au}^{0}\right)_{12}-\mathrm{E} 3 \cdot \mathrm{NH}_{2}\right\}$ (a) and $\left\{\left(\mathrm{Au}^{0}\right)_{57}-\mathrm{E} 5 . \mathrm{NH}_{2}\right\}$ (b) DSNPs, a typical SAED pattern of $\left\{\left(\mathrm{Au}^{0}\right)_{98}-\mathrm{E} 6 . \mathrm{NH}_{2}\right\}$ DSNPs (c), and an EDS spectrum of $\left\{\left(\mathrm{Au}^{0}\right)_{24}-\mathrm{E} 4 \cdot \mathrm{NH}_{2}\right\}$ DSNPs (d).

These larger Au nanoparticles will be very useful in cellular labelling and imaging studies.

All the synthesized Au DSNPs are highly polycrystalline as shown by both the high-resolution TEM images (figures 4(a) and (b)) and SAED patterns (e.g. figure 4(c)). In figures 4(a) and (b), typical crystal lattices for both single crystals and twin crystals of Au can be clearly seen. A typical SAED pattern of $\left\{\left(\mathrm{Au}^{0}\right)_{98}-\mathrm{E} 6 . \mathrm{NH}_{2}\right\}$ DSNPs (figure 4(c)) clearly shows the (111), (200), (220), and (311) rings, indicating the face-

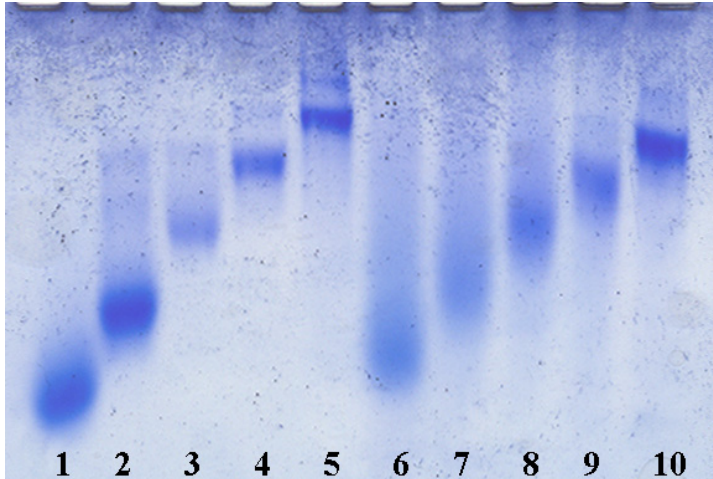

Figure 5. PAGE electropherograms of Au DSNPs and their corresponding dendrimer stabilizers. Lane 1, E2. $\mathrm{NH}_{2}$; lane 2, E3. $\mathrm{NH}_{2}$; lane 3, E4. $\mathrm{NH}_{2}$; lane 4, E5. $\mathrm{NH}_{2}$; lane 5, E6. $\mathrm{NH}_{2}$; lane 6, $\left\{\left(\mathrm{Au}^{0}\right)_{6}-\mathrm{E} 2 . \mathrm{NH}_{2}\right\} ;$ lane $7,\left\{\left(\mathrm{Au}^{0}\right)_{12}-\mathrm{E} 3 . \mathrm{NH}_{2}\right\} ;$ lane 8 , $\left\{\left(\mathrm{Au}^{0}\right)_{24}-\mathrm{E} 4 . \mathrm{NH}_{2}\right\} ;$ lane $9,\left\{\left(\mathrm{Au}^{0}\right)_{57}-\mathrm{E} 5 . \mathrm{NH}_{2}\right\} ;$ lane 10 , $\left\{\left(\mathrm{Au}^{0}\right)_{98}-\mathrm{E} 6 . \mathrm{NH}_{2}\right\}$.

centred-cubic (fcc) crystal structures. To our best knowledge, this is the first report regarding a high crystallite of Au DSNPs investigated by high-resolution TEM, although a crystallite of Pt DSNPs was reported recently by Jia and co-workers [44]. In order to confirm the composition of the formed Au DSNPs, EDS was collected for each Au DSNP samples. A typical EDS spectrum of $\left\{\left(\mathrm{Au}^{0}\right)_{24}-\mathrm{E} 4 . \mathrm{NH}_{2}\right\}$ DSNPs (figure 4(d)) clearly indicates the existence of $\mathrm{Au}$ elements.

Zeta potential measurements confirmed that all the synthesized Au DSNPs are positively charged (table 1), with zeta potentials ranging from 26.42 to $41.11 \mathrm{mV}$. This further indicates that after the formation of the hybrid nanostructures the terminal amines of dendrimers are still available to be protonated. The surface charge polarity of Au DSNPs is similar to the protonated corresponding dendrimers, which 
Characterization of crystalline dendrimer-stabilized gold nanoparticles

was further confirmed by polyacrylamide gel electrophoresis (PAGE) measurements. Shown in figure 5 are the PAGE electropherograms of both Au DSNPs and the corresponding dendrimer stabilizers. The Au DSNPs display very similar migration patterns to those of their respective dendrimer stabilizers. In some cases, the difference is that the $\mathrm{Au}$ DSNPs exhibit somewhat lower electrophoretic mobility than their corresponding dendrimer stabilizers due to their lower charge/mass ratios after 'loading' with Au nanocrystals. PAGE measurements verified the existence of dendrimers for each $\mathrm{Au}$ DSNP, because the Coomassie blue-stained bands of $\mathrm{Au}$ DSNPs are exclusively related to stained dendrimers. In contrast, commercial negatively charged citric-acid-protected Au NPs migrate reversely to the top side of the gel (toward the cathode) during electrophoresis under the reverse polarity. The PAGE results also indicate that the formed Au DSNPs are highly stable and both $\mathrm{Au}$ nanocrystals and dendrimers do not separate from each other during the electrophoresis at $\mathrm{pH}$ 8.3. Please note that we have also confirmed the existence of $\mathrm{Au}$ element in the respective PAGE bands of all Au DSNP samples by collecting the EDS spectra (data not shown) of the respective sliced gel films. Control gel samples without the Coomassie blue staining and gel bands of respective dendrimer stabilizers stained with Coomassie blue do not show the presence of Au signals.

\section{Conclusion}

In summary, $\mathrm{Au}$ DSNPs were prepared and stabilized using amine-terminated PAMAM dendrimers of different generations with the same molar ratios of terminal nitrogen ligands/gold atoms. For all synthesized Au DSNPs, UV-vis spectra show a plasmon peak at $525 \mathrm{~nm}$, while fluorescence spectroscopy demonstrates a strong emission at $458 \mathrm{~nm}$. All Au DSNPs were found to be highly polycrystalline with fcc crystal structures. The size of Au DSNPs decreases with the increase of the number of dendrimer generations. The formed Au DSNPs are positively charged, indicating that the protonation state of dendrimer stabilizers is not significantly influenced after the formation of the hybrid nanostructures. PAGE analysis further verified that the formed Au DSNPs are stable and integrated, and each Au DSNP can be considered as an entire entity. Our unpublished data show that the amine groups of dendrimers residing on the surface of Au DSNPs can be used to link biological ligands or can be modified with acetyl and hydroxyl groups, providing a promising strategy to modify them for biological sensing and cancer therapy.

\section{Acknowledgments}

This work is financially supported in part by the National Cancer Institute (NCI), National Institute of Health (NIH), under contract No NOI-CO-97111, and in part by the US Department of Energy (award No FG01-00NE22943). TRG thanks the UROP program for financial support. The $2010 \mathrm{~F}$ microscope used in the study was funded by the NSF through grant DMR-9871177. We thank Dr Rameshwer Shukla and Dr Xiangdong $\mathrm{Bi}$ for their valuable remarks.

\section{References}

[1] Tkachenko A G, Xie H, Coleman D, Glomm W, Ryan J, Anderson M F, Franzen S and Feldheim D L 2003 J. Am. Chem. Soc. 1254700

[2] Rosi N L and Mirkin C A 2005 Chem. Rev. 1051547

[3] Daniel M C and Astruc D 2004 Chem. Rev. 104293

[4] Parak W J, Gerion D, Pellegrino T, Zanchet D, Micheel C, Williams S C, Boudreau R, Le Gros M A, Larabell C A and Alivisatos A P 2003 Nanotechnology 14 R15

[5] Aubin M E, Morales D G and Hamad-Schifferli K 2005 Nano Lett. 5519

[6] Tkachenko A G, Xie H, Liu Y, Ryan J, Glomm W, Shipton M K, Franzen S and Feldheim D L 2004 Bioconjug. Chem. 15482

[7] El-Sayed I H, Huang X and El-Sayed M A 2005 Nano Lett. 5829

[8] Yang P H, Sun X, Chiu J F, Sun H and He Q Y 2005 Bioconjug. Chem. 16494

[9] Thanh N T K and Rosenzweig Z 2002 Anal. Chem. 741624

[10] Ho K C, Tsai P J, Lin Y S and Chen Y C 2004 Anal. Chem. 767162

[11] Balogh L and Tomalia D A 1998 J. Am. Chem. Soc. 1207355

[12] Esumi K, Suzuki A, Aihara N, Usui K and Torigoe K 1998 Langmuir 143157

[13] Crooks R M, Zhao M, Sun L, Chechik V and Yeung L K 2001 Acc. Chem. Res. 34181

[14] Zhao M, Sun L and Crooks R M 1998 J. Am. Chem. Soc. 1204877

[15] Tomalia D A, Baker H, Dewald J R, Hall M, Kallos G, Martin S, Roeck J, Ryder J and Smith P 1985 Polym. J. 17 117

[16] Tomalia D A, Baker H, Dewald J R, Hall M, Kallos G, Martin S, Roeck J, Ryder J and Smith P 1986 Macromolecules 192466

[17] Tomalia D A, Naylor A M and Goddard W A III 1990 Angew. Chem. Int. Edn Engl. 29138

[18] Zhao M and Crooks R M 1999 Angew. Chem. Int. Edn 38364

[19] Ispasoiu R G, Balogh L, Varnavski O P, Tomalia D A and Goodson T III 2000 J. Am. Chem. Soc. 12211005

[20] Ye J Y, Balogh L and Norris T B 2002 Appl. Phys. Lett. 801713

[21] Bielinska A, Eichman J D, Lee I, Baker J R Jr and Balogh L 2002 J. Nanopart. Res. 4395

[22] Balogh L, Bielinska A, Eichman J D, Valluzzi R, Lee I, Baker J R Jr, Lawrence T S and Khan M K 2002 Chim. Oggi/Chem. Today 2035

[23] He J A, Valluzzi R, Yang K, Dolukhanyan T, Sung C, Kumar J, Tripathy S K, Samuelson L, Balogh L and Tomalia D A 1999 Chem. Mater. 113268

[24] Esumi K, Akiyama S and Yoshimura T 2003 Langmuir 197679

[25] Esumi K 2003 Top. Curr. Chem. (Colloid Chem. II) 22731

[26] Esumi K 2004 Encyclopedia Nanosci. Nanotechnol. 2317

[27] Balogh L, Valluzzi R, Laverdure K S, Gido S P, Hagnauer G L and Tomalia D A 1999 J. Nanopart. Res. 1353

[28] Garcia M E, Baker L A and Crooks R M 1999 Anal. Chem. 71256

[29] Grohn F, Bauer B J, Akpalu Y A, Jackson C L and Amis E J 2000 Macromolecules 336042

[30] Manna A, Imae T, Aoi K, Okada M and Yogo T 2001 Chem. Mater. 131674

[31] Alvarez M M, Khoury J T, Schaaff T G, Shafigullin M N, Vezmer I and Whetten R L 1997 J. Phys. Chem. B 1013703

[32] Bohren C F and Huffman D R 1983 Absorption and Scattering of Light by Small Particles (New York: Wiley)

[33] Kreibig U and Vollmer M 1995 Optical Properties of Metal Clusters (Berlin: Springer)

[34] Lee W I, Bae Y and Bard A J 2004 J. Am. Chem. Soc 1268358 
[35] Sun X, Jiang X, Dong S and Wang E 2003 Macromol. Rapid Commun. 241024

[36] Zheng J, Petty J T and Dickson R M 2003 J. Am. Chem. Soc. 1257780

[37] Wang D and Imae T 2004 J. Am. Chem. Soc. 12613204

[38] Esumi K, Suzuki A, Yamahira A and Torigoe K 2000 Langmuir 162604

[39] Kim Y G, Oh S K and Crooks R M 2004 Chem. Mater. 16167
[40] Cushing B L, Kolesnichenko V L and O’Connor C J 2004 Chem. Rev. 1043893

[41] Hayakawa K, Yoshimura T and Esumi K 2003 Langmuir 195517

[42] Dean J A 1992 Lange's Handbook of Chemistry 14th edn (New York: McGraw-Hill)

[43] Lide D R 2002 CRC Handbook of Chemistry and Physics 83rd edn (New York: CRC Press LLC)

[44] Yang L, Luo Y, Jia X, Ji Y, You L, Zhou Q and Wei Y 2004 J. Phys. Chem. B 1081176 